\title{
Hierarchical Infrastructure for Large-Scale Distributed Privacy-Preserving Data Mining
}

\author{
Jinlong Wang, Congfu Xu, Huifeng Shen, and Yunhe Pan \\ Institute of Artificial Intelligence, Zhejiang University, \\ Hangzhou, 310027, China \\ zjupaper@yahoo.com \\ xucongfu@cs.zju.edu.cn \\ yaekee@hotmail.com \\ panyh@sun.zju.edu.cn
}

\begin{abstract}
Data Mining is often required to be performed among a number of groups of sites, where the precondition is that no privacy of any site should be leaked out to other sites. In this paper, a hierarchical infrastructure is proposed for large-scale distributed Privacy Preserving Data Mining (PPDM) utilizing a synergy between P2P and Grid. The proposed architecture is characterized with (1) its ability for preserving the privacy in data mining; (2) its ability for decentralized control; (3) its dynamic and scalable ability; (4) its global asynchrony and local communication ability. An algorithm is described to show how to process large-scale distributed PPDM based on the infrastructure. The remarks in the end show the effectiveness and advantages of the proposed infrastructure for large-scale distributed PPDM.
\end{abstract}

\section{Introduction}

Nowadays, data mining is one of the most important topics in large-scale distributed domains such as military, commerce and health-care etc. In the meanwhile, data privacy becomes a major concern that threatens the widespread deployment of data mining systems. This leads to a new sub-area of data mining privacy-preserving data mining (PPDM) [1] in large-scale distributed systems.

In this paper, we propose a hierarchical infrastructure for large-scale distributed PPDM. It synthesizes both Grid and P2P, and constructs a hierarchical architecture (super computers in the top level based on Grid [2], desktop computers and other pervasive computers in the bottom level, organized with P2P [3], which could thus help to ensure Grid scalability). In this way, it can achieve higher performance and satisfy the requirements of the reality better.

The remainder of the paper is organized as follows: section 2 describes the hierarchical infrastructure. Section 3 discusses the privacy preserving technology and cryptographic primitives. Section 4 gives a large-scale distributed PPDM algorithm based on the hierarchical infrastructure. Finally, we conclude with some remarks in the end. 


\section{Hierarchical Infrastructure}

The main purpose of the infrastructure is to supply a platform for large-scale distributed PPDM. The overall framework is shown as Fig1 below.

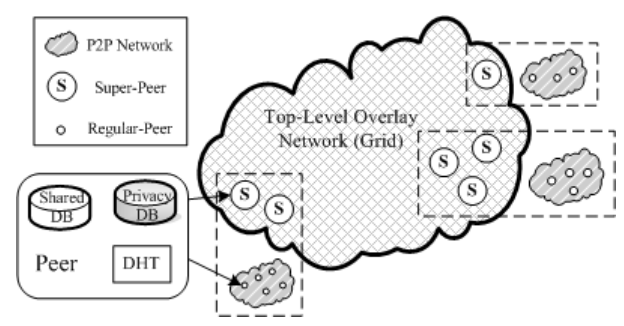

Fig. 1. Overall Framework of Architecture

Just as the scenario of Fig1, each peer (regular peer and super-peer) owns privacy database (PDB) which may not be open to other peers, shared database (SDB) which may be shared by other peers, and dynamic hash table (DHT) which is the table of route, indicating those peers the messages will be sent to. All of peers are organized into disjoint virtual groups (also called virtual organizers, VOs) by a series of protocols. Each VO maintains its own overlay network, including one or more super-peers, which are a subset of peers according to their computing resources and the data volume they store. These super-peers constitute a top-level overlay network (Grid [2]).

A VO may join and leave the system as a whole. In a VO, the numbers of peers can be dynamically changed by peers joining and leaving in any time. When a peer joins the system, it registers with one of existing peers. A peer can only have one connection to another peer higher in the hierarchy to register with, but be registered with many lower level peers. A peer communicates with its upper and lower peers through DHT. When a peer leaves the system, its lower peers must be informed to dynamically adjust the network topology to keep the hierarchical relations. The hierarchical protocol makes the larger scalable systems possible and can support the diversity of the cryptographical primitives.

Since the classification of group is according to the node distances and functions, there will be much more frequent communication among the same group than the one between groups. And, in a VO, we make use of asymmetrical bidirectional patterns to improve communication efficiency. Upward communication transfers all types of information, including system messages (peers joining or leaving) and data messages (data updating) to update up-peers, the downward communication transfers only system messages to adjust to the dynamic topology changes. In this way, the architecture can use the bandwidth more effectively, gain the advantages over Grid or P2P respectively, and thus suit a great variety of network environments better. 


\section{Privacy-Preserving and Cryptographic Primitives}

Based on the hierarchical architecture, multiplicity of privacy-preserving methods can be used. Literature [4] provides an overview of the new and rapidly emerging research area of PPDM. Making use of these methods, we can protect sensitive raw data and knowledge.

Within the different groups, we can utilize various cryptographic methods. Even in the same group, diversity of the security methods can also be sustained based on the demands.

In intra-group, the peers receive the messages below and in the same subset with them, but not directly mine the peers below and adjacent to them, which protects the privacy of the raw data. At the same time, the peers can modulate the messages sent to protect their sensitive knowledge. Based on the peer adjacent to it, we can shield the individual data sources to the peers not adjacent to it.

In the top level, the super-peers can use the cryptograph-based techniques to encrypt their private data. And none of peers are willing to disclose their own outputs to anyone else. With the cryptographic primitives, individuals and sources are protected.

\section{Large-Scale Distributed PPDM on the Architecture}

In this section, we apply the architecture to PPDM in large-scale distributed systems. In the interest of clarity, we consider the system where each group comprises a super-peer.

Just as the above scenario of Fig1, within each group, the super-peers are in charge of the hierarchical information of various sharing resources, each superpeer manages a range of peers, which may be the super-peer's neighbor or in the same subnet with it. Regular peers collaboratively mine data in P2P.

In $\mathrm{P} 2 \mathrm{P}$, for each peer, it receives the information from other peers, and then processes these information together with its PDB. After that, it will update its own PDB and transform the message sent to the SDB according to security strategies. Next, through its own DHT, it sends relevant fresh information in its SDB to other up-peers adjacent to it for furthering processing. In this way, the peer in the infrastructure consists of the knowledge mined from those peers below and in the same subnet with it. Layer upon layer, the super-peer comprises the up-to-date knowledge mined from the same group. Finally, in the top level, on the basis of the cryptograph-based techniques, we can process data mining with the Knowledge Grid [5] among the super-peers. The large-scale distributed PPDM algorithm based on the architecture is depicted as the algorithm 1 .

Within the hierarchical distributed architecture, the communication cost is evidently reduced to a large extent by never transmitting all data values but transmitting incremental and updated information. This approach does not require $\mathrm{CPU}$ and $\mathrm{I} / \mathrm{O}$ costs significantly higher than a similar approach and its communication may be lower. 


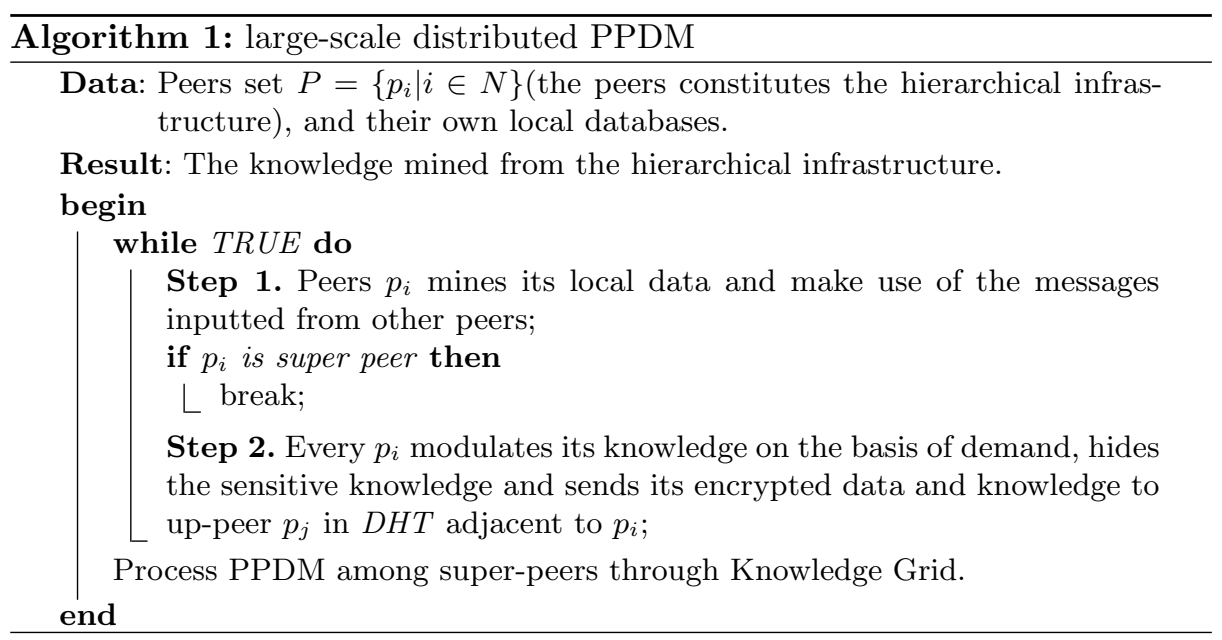

\section{Conclusions}

In this paper, we present a dynamic, hierarchical infrastructure for PPDM in large-scale loosely connected and constantly evolving environments. The bidirectional communication method can save more bandwidth. By adopting asynchrony communication pattern, our infrastructure avoids centralized control and improves the ability in suiting dynamic changes in current commercial network environment. In particular, by virtue of the hierarchial model based on P2PGrid, we can supply diversities of strategies for preserving both the privacy data sources and the sensitive knowledge. This property is especially important in industry, business and scientific applications.

\section{Acknowledgements}

This paper was supported by the Natural Science Foundation of China (No. 60402010) and the Advanced Research Project sponsored by China Defense Ministry (No. 413150804, 41101010207), and was partially supported by the Aerospace Research Foundation sponsored by China Aerospace Science and Industry Corporation (No. 2003-HT-ZJDX-13).

\section{References}

1. R. Agrawal, R. Srikant. Privacy-preserving data mining. SIGMOD 2000.

2. I. Foster, C. Kesselman, S. Tuecke. The Anatomy of the Grid: Enabling Scalable Virtual Organizations. International J. Supercomputer Applications, 15(3), 2001.

3. M. P. Singh. Peer-to-Peer Computing for information systems. AP2PC 2002.

4. V S. Verykios, E. Bertino, I N. Fovino, L P. Provenza, Y. Saygin, Y. Theodoridis. State-of-the-art in Privacy Preserving Data Mining. SIGMOD Record 2004, 33(1).

5. M. Cannataro, D. Talia. KNOWLEDGE GRID: An Architecture for Distributed Knowledge Discovery. Communications of the ACM, January 2003 46(1) 89-93. 